\title{
CULTES LOCAUX ET TRADITIONS HELLÉNISANTES DU PROCHE-ORIENT : À PROPOS DE LEUCOTHÉA ET DE MÉLICERTE
}

La publication du premier témoignage de piété à Leucothéa provenant du Proche-Orient remonte à 1886 . Depuis cette date, plusieurs trouvailles ont confirmé la popularité de la déesse grecque sur une vaste région allant de la Phénicie à la Décapole ${ }^{1}$. En 1993, le dossier syrien de Leucothéa s'est encore enrichi d'une dédicace associant cette divinité marine à son fils Mélicerte, sur l'autel d'Inkhil, dans le Hauran 2. Un texte découvert en 2004 à Haloua, sur le Mont Hermon, indique aujourd'hui que Mélicerte est aussi vénéré en territoire sidonien, non loin du village où Leucothéa possède son propre sanctuaire à l'époque romaine ${ }^{3}$. Ce témoignage invite à tirer parti de tous les éléments du dossier, dont plusieurs sont restés méconnus, pour identifier la Phénicie comme le foyer du culte de Leucothéa et de Mélicerte au Proche-Orient et pour montrer que la vénération de ces deux divinités par les populations locales, si elle trouve son origine dans l'interprétation grecque des dieux orientaux, n'y renvoie pas seulement à l'adoption de théonymes helléniques, mais aussi aux usages politiques des mythes grecs dans les cités voisines de Tyr et de Sidon aux époques hellénistique et romaine.

\section{Leucothéa et Mélicerte en Phénicie}

Au Proche-Orient, les témoignages de dévotion adressés à Leucothéa et à son fils Mélicerte sont pour la plupart des inscriptions provenant de la Phénicie côtière (Tyr, Béryte), de la Békaa (Kfar Zabad), du Mont Hermon (Haloua, Rakhlé, Ain el-

1. Je remercie Pierre-Louis Gatier et Jean-Baptiste Yon (HISOMA, Maison de l'Orient, Lyon) pour leur lecture attentive et leurs remarques critiques.

2. SARTRE 1993, p. $52, \mathrm{n}^{\circ} 1$.

3. Aliquot 2004. 
Burj), du Hauran (Inkhil) et de la Décapole (Gérasa, Tel Jezreel sur le territoire de Scythopolis) ${ }^{4}$. Toutes n'attestent pas l'existence de sanctuaires consacrés à l'une ou à l'autre des deux divinités : ainsi, les inscriptions de Tyr, Béryte, Haloua, Kfar Zabad, Inkhil, Tel Jezreel et Gérasa fournissent uniquement des informations sur la propagation de leur culte. Jusqu'à présent, on ne connaît que deux lieux saints dédiés à Leucothéa dans la région : il s'agit de sanctuaires montagnards situés sur l'Hermon et le seul qui ait été localisé avec certitude se trouve à Rakhlé, en territoire sidonien. Cependant d'autres témoignages, nouveaux ou incomplètement analysés ou encore méconnus, complètent le dossier syrien de la déesse blanche de l'écume, nommée Ino avant sa divinisation, et de son fils : outre l'inscription de Haloua, il s'agit d'un passage de Lucien de Samosate sur Ino à Sidon, d'une dédicace inédite de Béryte et de deux textes de Tyr et de Deir el-Qalaa, que je propose de réviser. L'étude de ces documents avait amené certains auteurs à souligner, à la suite d'Henri Seyrig, les similitudes entre les cultes du Mont Hermon et ceux de la région correspondant précisément à la zone d'extension des dédicaces à Leucothéa, de la côte phénicienne à la Décapole ${ }^{5}$. Aujourd'hui, elle permet aussi de considérer la Phénicie comme le foyer à partir duquel la vénération de Leucothéa et de Mélicerte s'est propagée selon des modalités diverses à partir de l'époque hellénistique.

Bien que le Mont Hermon soit la seule région du Proche-Orient où l'on connaisse des sanctuaires consacrés à Leucothéa, l'invocation de cette divinité et de son fils y est vraisemblablement tardive. Aux confins du territoire de Sidon, en 60 p.C., la plus ancienne inscription datée de Rakhlé mentionne encore la déesse locale de manière anonyme, en accolant à son nom celui du fondateur du culte, Moithos ${ }^{6}$. Ce n'est qu'ensuite, au $\mathrm{II}^{\mathrm{e}}$ et au $\mathrm{III}^{\mathrm{e}}$ siècle $p . C$., que la même divinité est nommée Leucothéa et considérée banalement comme la grande déesse tutélaire du village ${ }^{7}$. Non loin de là, le libellé de l'ordonnance divine de Haloua révèle que le culte de Mélicerte fait lui aussi l'objet d'une adaptation peu conforme à la tradition hellénique et sans doute aussi tardive qu'à Rakhlé, puisque le jeune fils d'Ino y est présenté comme un dieu et assimilé au messager d'une divinité

4. À la collection des textes réunis par SARTRE 1993 pour composer le dossier syrien de Leucothéa et de Mélicerte, on ajoutera la dédicace de provenance incertaine publiée par GATIER 1994, p. 776-779 (peut-être de Rakhlé), l'inscription de Tel Jezreel éditée par Porat 1997, celle de Haloua et un inédit de Beyrouth. J'ajoute encore que, d'après Pausanias, Description de la Grèce 2, 1, 8-9, l'une des images des divinités marines exposées dans le sanctuaire de la Néréide Doto à Gabala représente Ino accompagnée de Bellérophon.

5. Seyrig 1963, p. 26 (Seyrig 1966, p. 128); cf. Gatier 1982, p. 275.

6. SARTre 1993, p. 55-57, nº 4, cf. GATIER, Bull. épigr. 1994, 636 ( $\theta \varepsilon \hat{\alpha} \varsigma$ Mot $\theta o v)$.

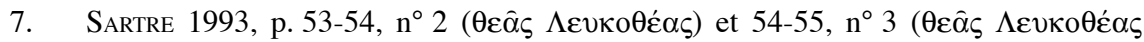
P $\alpha \chi \lambda \alpha \varsigma)$. La grande déesse de Rakhlé reste parfois anonyme. Cf. CleRMONT-GANNEAU

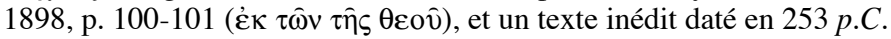


suprême de la montagne ${ }^{8}$. L'adaptation d'un culte étranger à un contexte local est ici manifeste dans la mention peu commune du dieu sous le nom de Mélicerte plutôt que sous celui, plus classique et conforme aux données mythologiques, de Palémon. C'est en effet sous ce dernier nom que le fils de Leucothéa divinisé est habituellement invoqué, en particulier à l'Isthme de Corinthe, où se trouve son principal sanctuaire 9 . À l'exception de quelques textes magiques, le dieu n'est guère appelé Mélicerte qu'à Haloua, sur l'Hermon, et à Inkhil, dans le Hauran voisin ${ }^{10}$.

En plus des monuments et des inscriptions de Rakhlé et de Haloua, localités du territoire sidonien, un passage de Lucien de Samosate me paraît renvoyer à la présence d'Ino à Sidon même. Dans les Dialogues des dieux, Hermès, éreinté, conte à sa mère, la nymphe Maia, les tribulations que Zeus lui a imposées : « À l'instant même, j' arrive de chez la fille de Cadmos à Sidon, où il m'a envoyé pour voir ce que faisait la petite ; et sans me laisser le temps de souffler, il m'a envoyé à nouveau à Argos pour veiller sur Danaé ; "Ensuite", dit-il, "tu iras de là en Béotie et tu jetteras un coup d'œil sur Antiope en route." Mais je suis déjà complètement épuisé » ${ }^{11}$. Matthew D. Macleod, l'éditeur de Lucien, suppose qu'au début de ce

8. Aliquot 2004. Le texte commémore l'affichage public de la prescription d'un dieu

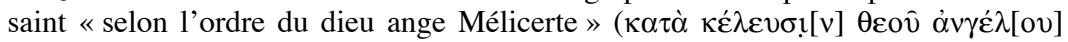

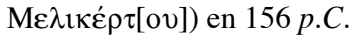

9. En dernier lieu Piérart 1998 et GebHARd 2005. Sur la mosaïque d'Agreus, dans la grande salle des bains $\mathrm{E}$ à Antioche ( $\mathrm{V}^{\mathrm{e}}$ siècle $p . C$.), le dieu porte le nom classique $\Pi \alpha \lambda \varepsilon \dot{\mu} \mu \omega v$, bien qu'il soit représenté de manière originale sous la forme d'un triton à la syrinx emportant la Néréide Actaé. Voir BALTY 1981.

10. SARTRE 1993, p. 52, n 1 , sur le dé et sur la moulure inférieure d'un petit autel retrouvé

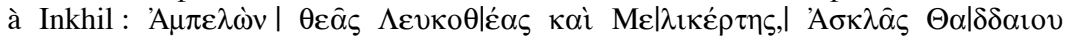

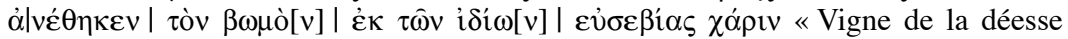
Leucothéa et de Mélicerte. Asclas fils de Thaddaios a consacré l'autel sur ses propres

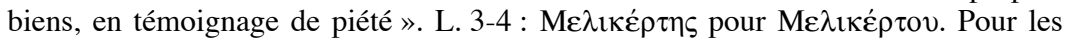
textes magiques invoquant Mélicerte, cf. $S E G$ 41, 1844. J'observe qu'à l'époque hellénistique, c'est Mélicerte et non Palémon que le poète et philosophe Philodème de Gadara prie de lui permettre de voyager sans encombre. Voir Anthologie palatine 6, 349 (éd. et trad. WALTz 1960, p. 167) : « Mélicerte fils d'Ino, et toi, Leucothéa aux yeux pairs, reine de la mer, déesse secourable, et vous, chœurs des Néréides, ondes, Poséidon, Zéphyr de Thrace, le plus doux des vents, soyez-moi propices et portezmoi sans dommage, à travers la vaste étendue des flots, jusqu'au doux rivage du Pirée. »

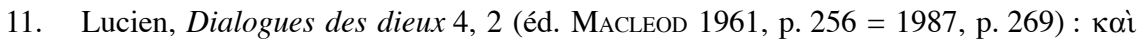

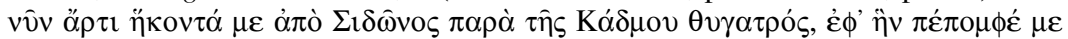

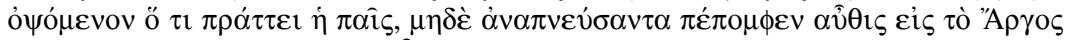

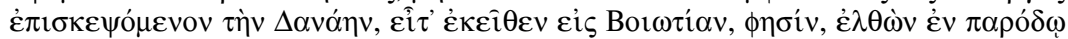

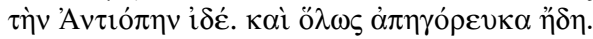


passage, le satiriste évoque Europe en l'identifiant à tort à la fille de Cadmos ${ }^{12}$. Tout en reconnaissant l'invraisemblance d'une telle erreur de la part de Lucien, il refuse d'admettre qu'il est question d'Ino à Sidon. Or, ce doute n'est pas fondé, et ce pour trois raisons. La première, mentionnée par Macleod lui-même, est qu'ailleurs le même Lucien présente Europe comme la fille d'Agénor, de manière tout à fait

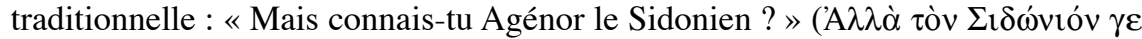

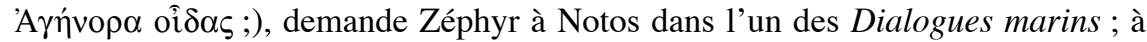

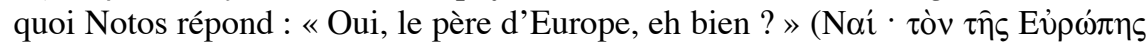

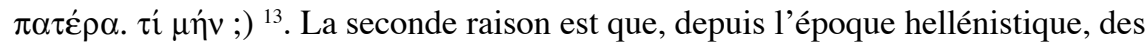
Sidoniens rendent hommage à Ino et à son fils : le poète sidonien Antipatros est l'auteur d'une épigramme votive en leur honneur ${ }^{14}$; on sait par ailleurs que les Sidoniens d'Athènes fréquentent alors le sanctuaire sans temple de l'Ilissos, où Palémon est soit le parèdre, soit l'alter ego de Pancratès et d'Héraclès dès la fin du $\mathrm{IV}^{\mathrm{e}}$ siècle $a . C .{ }^{15}$. La troisième et dernière raison est que le culte d'Ino-Leucothéa est bien attesté sur le territoire de Sidon à l'époque où écrit Lucien : la présence en ville de la déesse au II $^{\mathrm{e}}$ siècle $p$.C. est donc vraisemblable et le satiriste a pu s'y référer. Le passage cité pourrait révéler une fois de plus la connaissance intime que Lucien avait des cultes et des traditions des cités de l'Orient méditerranéen ${ }^{16}$ :

12. MACleOD 1961, p. 256-257 n. 2. L'idée remonte à François Guyet, qui corrige arbitrairement $\theta v \gamma \alpha \tau \rho o ́ \varsigma$ par $\alpha \dot{\delta} \delta \lambda \phi \eta \hat{\varsigma}$ dans une note de l'édition de Lucien publiée par Johannes Graevius et parue à Amsterdam en 1687. Si Guyet n'est plus suivi dans la modification du texte, sa proposition subsiste, soit qu'on en doute comme TALBOT 1912, p. 98 n. 1, soit qu'on l'adopte comme LightFoot 2003, p. 302. ChAmBry 1933, p. 150 et 508 n. 115 , se contente d'identifier la fille de Cadmos à Ino, à juste titre mais sans commentaire.

13. Lucien, Dialogues marins 15,1 .

14. Antipatros de Sidon, dans Anthologie palatine 6, 223 (éd. et trad. WaLtz 1960, p. 117) : «Ce débris mutilé d'une scolopendre, monstre qui sillonne les flots, gisait sur le sable du rivage, long de huit brasses, souillé en dessous par l'écume et tout déchiré sur les récifs de la mer ; Hermonax l'y a découvert, alors qu'en exerçant son métier de pêcheur il retirait de la mer son filet rempli de poissons ; et c'est cette trouvaille qu'il a consacrée à Ino et à son fils Palémon, dédiant aux divinités marines une merveille marine ».

15. Pour les trouvailles épigraphiques du sanctuaire de l'Ilissos, J. \& L. RoBert, Bull. épigr. 1959, 124, à propos de leur publication préliminaire. L'ensemble de la documentation relative aux cultes locaux est aujourd'hui réunie et étudiée par VIKELA 1994.

16. Le livre de Jones 1986 analyse systématiquement l'actualité des écrits de Lucien, dans le prolongement des réflexions de ROBERT 1980, p. 393-436 (ch. 18 « Lucien en son temps »). Ce dernier rappelle opportunément (p. 404) un exemple syrien illustrant la fiabilité de l'information transmise par le prosateur, d'après une inscription d'Antioche publiée par Paul Perdrizet en 1903 et absente des IGLS : il s'agit d'un vers apotropaïque gravé à l'époque impériale pour écarter la peste, qui 
sur le mode de la plaisanterie, le périple d'Hermès ferait ainsi le tour des relations mythiques que Sidon se prévalait d'entretenir depuis l'époque hellénistique avec Argos, la patrie d'Agénor, et avec la Béotie, terre d'élection de Cadmos, comme on le verra plus loin.

Leucothéa fait aussi partie des grands dieux de Tyr à l'époque romaine. Selon une dédicace publiée par René Mouterde en 1962, son culte est placé à l'époque sévérienne sous la responsabilité d'un prêtre de la Bonne Fortune, Lucius Septimius Diodoros, qui cumule cette charge avec l'archiprêtrise perpétuelle du Seigneur Héraclès, interprétation grecque du grand dieu poliade Melqart. Le même texte montre que Leucothéa est également associée à une divinité oraculaire dont le nom est devenu illisible ${ }^{17}$. C'est celui de Mélicerte que je propose de restituer

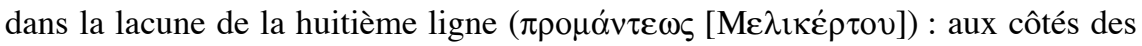
grands dieux de la cité, parmi lesquels figure sa mère Leucothéa, Mélicerte a pu endosser à Tyr le rôle qu'on lui attribue à Haloua, celui du jeune porte-parole de la volonté divine ${ }^{18}$. L'association du fils de Leucothéa au dieu tyrien ne serait pas sans rappeler celle de Palémon à Héraclès, bien attestée depuis l'époque classique ${ }^{19}$.

Enfin, la liste des lieux de la Phénicie où Leucothéa est honorée comprend la colonie romaine de Béryte et deux sites cultuels de son arrière-pays montagneux, Kfar Zabad et peut-être Deir el-Qalaa. À Béryte même, un citoyen romain consacre un autel en latin aux dieux de la triade héliopolitaine et à la déesse Leucothéa pour son propre salut et pour celui des siens, en exécution d'un vœu ${ }^{20}$. Sur le Jabal

reproduit un oracle d'Alexandre d'Abonouteichos littéralement cité au paragraphe 36 de l'opuscule intitulé Alexandre, ou le faux prophète. J'ajoute que Michon 1905, p. 578, republiant le texte d'Antioche, en signale l'entrée dans les collections du musée du Louvre.

17. Mouterde, dans ChéHab 1962, p. 17-19, avec photographie pl. 5, 1 (SARTRE 1993, p. 58, nº 6), cf. ROBERT, Bull. épigr. 1964, 499 et BONNET 1988, p. 63.

18. L'usage du substantif $\pi \rho o ́ \mu \alpha v \tau \iota \varsigma$ pour une divinité est attesté par exemple chez Euripide, Ion 681-682, à propos d'Apollon : « ô devin, fils de Létô, que nous annonce

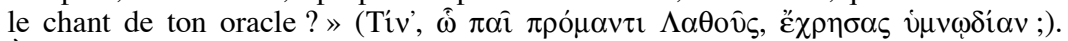
À l'époque romaine, le synonyme $\mu$ óv $\tau ı \varsigma$ est utilisé avec la même acception : au

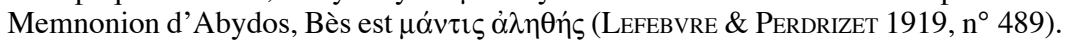
Pour sa part, Chuvin 1991, 229 et 237 n. 59, suggère d'identifier le devin tyrien à la fois à Iolaos, neveu d'Héraclès, et au dieu ange de Milkashtart des inscriptions phéniciennes de Maachouq et d'Oumm el-Amed, mais rien ne semble justifier une telle proposition.

19. Vikela \& Vollkommer 1992 (représentations iconographiques); Vikela 1994 (reliefs du sanctuaire athénien de l'Ilissos); IG 7, 2874 (dédicace de Coronée à Héraclès, à Palémon et à la cité) ; Plaute, Rudens 160-161 (sed o Palemon, sancte Neptuni comes, qui Herculis socius esse diceris).

20. Inscription inédite découverte en 2005 dans le centre-ville de Beyrouth. 
Terbol, au sud de la Békaa libanaise, un socle ou un cippe votif découvert dans les ruines de l'un des deux temples de Kfar Zabad porte exceptionnellement le nom et l'image de la déesse sur sa face principale : tout comme la Dea Syria, Leucothéa prend l'apparence d'une divinité composite flanquée de lions, dont l'aspect rappelle autant celui de la Fortune que celui d'une Atargatis ou d'une Allat ; le monument est explicitement dédié à Leucothéa en grec et au Jupiter héliopolitain en latin, selon une association qui se conçoit sans difficulté dans une localité située à mi-chemin entre Héliopolis-Baalbek et le Mont Hermon ${ }^{21}$. À Deir el-Qalaa, sur le versant maritime du Mont Liban, un autel porte une dédicace à Mater Matuta ${ }^{22}$. Il est bien connu que, sous l'Empire, les Romains identifient couramment Leucothéa à cette déesse italique de l'aurore et de la naissance, protectrice des matrones : selon les poètes et les mythographes, Mater Matuta, qui, à Rome, possède un temple sur le Forum Boarium, à côté du port de la Ville, n'est autre qu'Ino-Leucothéa, venue aborder là après sa divinisation ${ }^{23}$. Pour autant, Jean-Paul Rey-Coquais estime qu'à Deir el-Qalaa, il faudrait la distinguer de Leucothéa ${ }^{24}$. De la même manière, Fergus Millar affirme que la dédicante, Flavia Nicolais Saddane, adhère à un culte importé et spécifiquement romain ${ }^{25}$. Pour sa part, Corinne Bonnet privilégie de manière peu convaincante l'hypothèse d'une assimilation à Astarté ${ }^{26}$. Je pense que deux interprétations peuvent se combiner pour rendre compte de l'invocation de Mater Matuta dans l'arrière-pays de Béryte : d'une part, il n'est pas exclu qu'un authentique culte romain soit importé ; mais, d'autre part, tout comme chez les

21. Ghadban 1981, p. 153 (SARTRe 1993, p. 59, n 8) : sur la corniche, I(ovi) O(ptimo) M(aximo) H(eliopolitano); sur la face principale du dé, $\Lambda \varepsilon v \kappa \alpha \theta \varepsilon ́ \alpha$. L. 2 : Sartre transcrit par inadvertance $\Lambda \varepsilon v \kappa o \theta \varepsilon ́ \alpha$. Le dé du monument porte sur trois faces des sculptures en haut-relief. Sur la face antérieure, une niche cintrée abrite l'image de la déesse, debout et vêtue d'une tunique et d'un manteau. La tête est mutilée, l'avantbras droit brisé ; une masse informe est visible sur l'épaule, ce qui indique que le bras gauche tenait autrefois un objet, peut-être une corne d'abondance. Sur les parois latérales du dé, la déesse est flanquée de deux lions de face, également mutilés. Ainsi représentée et accompagnée, Leucothéa ressemble formellement à Atargatis et à Allat.

22. CIL 3 Suppl., 6680 : Matri Matutae | Flavia T(iti) f(ilia) Nicolais I Saddane, L(ucii) Antistii | Veteris, ex responso | deae Iunonis aram | fecit dedicavitque « À Mater Matuta, Flavia Nicolais Saddane, fille de Titus, (épouse) de Lucius Antistius Vetus, a fait (faire) et a consacré cet autel conformément à une réponse oraculaire de la déesse Junon ».

23. Voir les références textuelles réunies par BonNet 1986, p. 67, et par Pí́RART 1998, p. 104-105.

24. Rey-CoQuais 1999, p. 620.

25. Millar 1993, p. 281.

26. BONNET 1991, p. 78. 
auteurs grecs et latins, les théonymes Mater Matuta et $\Lambda \varepsilon v \kappa o \theta \varepsilon ́ \alpha$ peuvent aussi être interchangeables pour la dédicante, qui fait partie de l'élite sociale de la colonie romaine ${ }^{27}$. La dédicace de Deir el-Qalaa suggère que, sur les territoires coloniaux de Béryte et d'Héliopolis, lorsqu'ils écrivent le nom de la déesse en latin, les fidèles la nomment éventuellement Mater Matuta. En revanche, ils lui donnent le nom de Leucothéa lorsqu'ils rédigent une dédicace en grec, comme sur le cippe votif de Kfar Zabad. La corrélation entre le recours à des théonymes grecs ou latins et la langue dans laquelle un acte religieux est rédigé est assez bien attestée par ailleurs, notamment pour le Jupiter héliopolitain (en latin IOMH sur le monument de Kfar Zabad), pour rendre plausible cette hypothèse : à Deir elQalaa, Mater Matuta serait à la fois la protectrice des matrones et l'interprétation romaine de la déesse vénérée ailleurs sous un nom grec.

\section{Un culte multiforme}

Pour expliquer le succès régional de Leucothéa et de Mélicerte à l'époque romaine, on insiste surtout sur l'interprétation grecque des dieux locaux, non sans argument. Selon l'opinion formulée par Clermont-Ganneau il y a plus d'un siècle et unanimement adoptée depuis, le nom de Leucothéa, de Tyr à Gérasa, remplacerait celui de grandes divinités orientales avec lesquelles la déesse grecque a des points communs ${ }^{28}$. Ainsi, le récit du saut dans la mer d'Ino et de son jeune fils, qui précède leur divinisation, rappelle certains épisodes de l'histoire d'Astarté, de Dercéto ou d'Atargatis. Aujourd'hui, cette proposition reste vraisemblable, compte tenu des caractères originaux et de l'importance de Leucothéa et de Mélicerte en milieu rural. Il n'en reste pas moins difficile de déterminer quelles caractéristiques de la déesse grecque et de son fils peuvent séduire les fidèles au point qu'ils adoptent leurs noms dès l'époque hellénistique et qu'ils vont peut-être jusqu'à invoquer ces dieux grecs eux-mêmes, en particulier en Phénicie. En effet, on est toujours fondé à postuler que l'hellénisation de la nomenclature divine procède d'une construction propre aux localités où Leucothéa et Mélicerte sont attestés, parce que leur culte revêt partout des caractères spécifiques à l'époque romaine,

27. L'époux de Flavia Nicolais Saddane est apparenté à la noble famille romaine des Antistii Vetii, dont plusieurs membres ont accédé au consulat. Cf. le commentaire à CIL 3 Suppl., 6680.

28. Clermont-Ganneau 1898, p. 68-69; Perdrizet 1932, p. 207-209; Gatier 1982, p. 275 ; HaJJAR 1985, p. 231 ; BonNet 1986, p. 64-67 ; BONNET 1988, p. 66 ; HAJJAR 1990, p. 2543 ; BonNet 1991, p. 78 ; SARTRE 1993, p. 60-61; GatIER 1994, p. 778 ; BonNet 1996, p. 42 ; BonNet 1997, p. 92 ; SARTRe 2003, p. 889 et 902. J'ai moi-même suivi l'avis de Maurice Sartre sur ce point dans Aliquot 2002, p. 238. De manière pertinente, LighTFOOT 2003, p. 70-72, relève les faiblesses de l'opinion commune, qui repose essentiellement sur les analogies entre les mythes de Leucothéa et des déesses proche-orientales, mais elle n'en tire aucune conclusion. 
en milieu rural comme en milieu urbain : alors qu'en ville et dans la Békaa, la déesse marine et le jeune dieu ne sont vraisemblablement que les parèdres mineurs occasionnels du Zeus de Gérasa et du Jupiter héliopolitain sans que leur culte ait un rapport direct avec celui de ces grands dieux poliades, Leucothéa devient la grande déesse tutélaire de communautés villageoises sur l'Hermon et Mélicerte le messager du dieu suprême.

D'autres arguments en apparence plus triviaux que ceux que l'on tire de l'étude des mythes pourraient encore justifier l'hellénisation de la nomenclature divine en milieu rural. Dans les anthroponymes relevés sur le Mont Hermon, l'apparition récurrente de l'élément pansémitique ' $n(t)$ (Anat) laisse supposer que ce théonyme est remplacé localement par celui d'Ino, en vertu de la proximité phonétique entre ' $n(t)$ et 'Iv $\omega^{29}$. Quant à l'invocation de Mélicerte sur l'Hermon et dans le Hauran, elle pourrait aussi résulter de l'intention de débarbariser un culte local par un jeu semblable sur des analogies phonétiques entre un théonyme grec et un théonyme sémitique. À ce propos, il est vrai que tout rapprochement

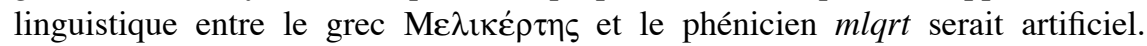
C'est pourquoi Maurice Sartre, l'éditeur du texte d'Inkhil, qui livre la première attestation connue de Mélicerte au Proche-Orient, me semble nier à juste titre que cette divinité soit assimilable au dieu tyrien Melqart ${ }^{30}$. Pour autant, même si rien ne permet d'identifier le jeune dieu oriental correspondant au fils de Leucothéa, il n'est pas exclu, à mon avis, que l'usage du théonyme grec Mع favorisé en Orient par la ressemblance entre ce nom et le phénicien mlqrt, une analyse philologique en bonne et due forme ne pouvant guère être exigée des populations locales.

Sur le Mont Hermon, les fidèles ne se contentent d'ailleurs sans doute pas de rapprochements aussi approximatifs pour attribuer des noms grecs à leurs dieux. Il est probable qu'ils s'appuient aussi sur des analogies entre le culte de ces derniers et celui de Leucothéa et de son fils. En reprenant la publication et le commentaire de la dédicace d'Ain el-Burj, qui commémore la réalisation de travaux dans le second sanctuaire hermonien de Leucothéa, celui de Segeira, j'ai rappelé naguère le caractère funéraire de l'expression relative à l'apothéose d'un parent du dédicant, qui impliquerait l'existence d'un monument associé au lieu saint où le défunt aurait reçu des honneurs posthumes ${ }^{31}$. Ni la localisation, ni l'aspect du sanctuaire

29. Selon la conjecture de GATIER 1994, p. 778 n. 14, à propos des anthroponymes théophores tels $\mathrm{A} \beta 1 \delta \alpha \alpha v \alpha \varsigma, A \mu \alpha \theta \alpha v \alpha$ et $\mathrm{Z} \alpha \beta \delta \alpha \alpha v \alpha \varsigma$, attestés principalement sur l'Hermon et dans l'Antiliban.

30. SARTRe 1993, p. 62, suivant BonNet 1986, p. 59-63, dont West 1997, p. 58, ignore les objections.

31. Aliquot 2002. Le sanctuaire de Segeira n'est pas localisable avec précision. La découverte de la dédicace qui en révèle l'existence à Ain el-Burj, sur l'Hermon oriental, laisse supposer qu'il ne se trouve pas sur le territoire de Sidon, mais plutôt sur celui de Damas. 
de Segeira ne sont précisément connus, mais il est probable que ce lieu saint soit entouré d'installations funéraires telles qu'on en repère à proximité de la plupart des temples romains de la région. Or, à l'époque de la dédicace d'Ain el-Burj,

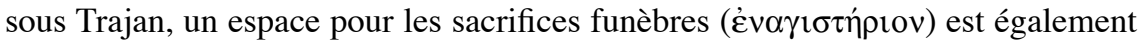
réservé dans le sanctuaire de l'Isthme de Corinthe consacré à Palémon ${ }^{32}$. Quel que soit le rapport entre ces rites et les cérémonies associées aux sanctuaires hermoniens, on peut supposer que les populations locales ont été séduites par les connotations funèbres du culte rendu au fils de Leucothéa divinisé, soit en Grèce, soit en Phénicie.

La recherche des analogies mythologiques, nominales et fonctionnelles entre les traditions grecques et orientales est intéressante en ce qu'elle conduit à souligner le caractère multiforme du culte de Leucothéa et de Mélicerte au ProcheOrient. Cependant, elle ne permet pas de rendre compte du caractère régional de ce culte ni de sa propagation à partir d'un foyer phénicien. À cet effet, l'étude doit être complétée par la mise en évidence des principes politiques qui sous-tendent l'invocation des dieux grecs sur le territoire des deux villes où ils connaissent leur succès le plus grand ou le plus précoce : Tyr et Sidon.

\section{Le mythe de Leucothéa au service de Tyr et de Sidon}

Selon la version la plus répandue du mythe de Leucothéa, la déesse est à l'origine Ino, fille du fondateur de Thèbes, le Phénicien Cadmos ${ }^{33}$. Elle épouse le roi Athamas, avec qui elle a deux fils, Mélicerte et Léarchos. Tous deux sont élevés avec le petit Dionysos, fils des amours adultères de Zeus et de Sémélè, ce qui suscitera la colère d'Héra envers Athamas et Ino. Jalouse des enfants issus du premier mariage d'Athamas, Phrixos et Hellè, Ino obtient par un stratagème qu'ils soient sacrifiés, mais ces derniers parviennent à s'enfuir sur un bélier dont la toison dorée sera l'objet de la quête de Jason et des Argonautes. Plus tard, frappé de folie par Héra, Athamas tue Léarchos. Ino, pour sauver Mélicerte de son père, l'entraîne alors dans la mer en s'y jetant elle-même. Tandis qu'Ino devient la déesse Leucothéa, Mélicerte divinisé devient Palémon, protecteur des ports et des marins.

Le cycle béotien auquel ce mythe se rapporte est intégré dans les traditions hellénisantes de Tyr à l'époque romaine. La tradition qui lie la cité phénicienne à la famille de Cadmos est aussi ancienne qu'indiscutable ${ }^{34}$. Lorsqu'il affirme que

32. Voir Piérart 1998, p. 97-99 et 105-106 et Gebhard 2005, p. 189-203.

33. BonNEt 1986, p. 53-56, résume les différentes versions du mythe.

34. Pour Hérodote, Histoires 2, 47 et 4, 45, Cadmos et Europe, les enfants du Phénicien Agénor, sont Tyriens. Pour sa part, Pausanias, Périégèse 5, 25, 12, insiste sur l'origine tyrienne d'une partie des colons phéniciens établis à Thasos par un fils d'Agénor. 
« les Tyriens croient Dionysos de chez eux, puisqu'ils chantent aussi la légende de Cadmos », le prosateur alexandrin Achille Tatius fait certainement référence à une légende locale qui désigne Tyr comme la cité où Dionysos a été élevé par sa nourrice Ino ${ }^{35}$. Les types monétaires tyriens qui représentent les aventures de Cadmos et d'Europe $\mathrm{au} \mathrm{II}^{\mathrm{e}}$ et $\mathrm{au} \mathrm{III}^{\mathrm{e}}$ siècle p.C. confirment l'assertion d'Achille Tatius et tendent à montrer que Thèbes n'est qu'une colonie de la cité phénicienne ${ }^{36}$. On peut supposer que les traditions locales intègrent aussi la légende des Argonautes car, chez Nonnos de Panopolis, Tyr reçoit l'épithète homérique de la nef Argo, $\pi \alpha \sigma u \mu \varepsilon \dot{\lambda} \sigma v \sigma \alpha$ «célébrée de tous » ${ }^{37}$. L'apparition de Leucothéa et de Mélicerte aux côtés d'Héraclès, dans l'inscription tyrienne restituée plus haut, montrerait à nouveau que les mythes grecs ont été adaptés localement. De la même manière, Europe est mise au service du grand dieu poliade : la légende de monnaies frappées à Tyr au $\mathrm{III}^{\mathrm{e}}$ siècle $p . C$. lui attribue un rôle dans le sanctuaire d'Héraclès en la

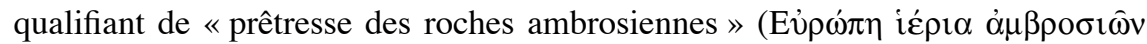
$\pi \varepsilon \tau \rho \hat{\omega} v)^{38}$.

Les épisodes mythiques relatifs à la lignée cadméenne font aussi partie des traditions hellénisantes de Sidon. La cité frappe des monnaies au type d'Europe sur le taureau pendant plus de quatre siècles, de la fin du $\mathrm{III}^{\mathrm{e}}$ siècle $a$.C. au début du III $^{\mathrm{e}}$ siècle p.C., avant d'adopter le type de Cadmos à partir de Trajan ${ }^{39}$, puis, sous les Sévères, celui qui représente les compagnons de Jason en train de souquer sur l'Argo ${ }^{40}$. De la part des Sidoniens, le choix de ces types monétaires apparaît comme un moyen d'affirmer l'hellénisme de la cité phénicienne qui se présentait

Dans son traité De l'abstinence 4, 19, 2, le Tyrien Porphyre cite un vers d'une pièce perdue d'Euripide, Les Crétois, où Europe serait Tyrienne, mais l'établissement du texte pose autant de problèmes aux éditeurs de Porphyre (PAtillon \& SEgonds 1995, p. 32 et 91) qu'à ceux d'Euripide (KanNicht 2004, p. 505).

35. Achille Tatius, Leucippé et Clitophon 2, 2,1 (éd. Garnaud 1991, p. 32) : Tòv rà

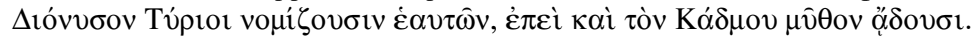

36. Chuvin 1991, p. 245-249.

37. Nonnos de Panopolis, Dionysiaques 40, 351.

38. MOUTERDE 1942-1943, p. 77-79, fig. 10. Sur les roches ambrosiennes, qui font partie des symboles du sanctuaire d'Héraclès à Tyr, voir WILL 1950-1951 (WILL 1995b, p. 243-255) et, en dernier lieu, le dossier textuel et numismatique compilé par NASTER 1986.

39. Chuvin 1991, p. 246.

40. Blatter 1984, nº 8 (monnaie de Sidon au type des Argonautes, identifiés par la

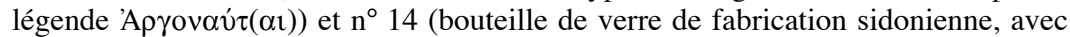
Jason et la Toison d'Or d'un côté et l'Argo de l'autre). L'Argo figure aussi à la même époque sur les monnaies de la cité de Césarée-Paneas, au pied du Mont Hermon. Voir MeSHORER 1984-1985, p. 46-47 et pl. 15. 
déjà comme la métropole de Thèbes vers $200 a . C .{ }^{41}$; il sert aussi à contester aux Tyriens le droit d'accaparer les mythes grecs relatifs à Cadmos et à sa prestigieuse lignée.

La thématique cadméenne qui envahit la numismatique des deux villes témoigne ainsi des trésors d'ingéniosité que dépensent les citoyens de Tyr et de Sidon depuis l'époque hellénistique pour afficher leur préséance : le titre de métropole de Thèbes ne lui suffisant pas, Sidon se dit métropole de Tyr, ce à quoi Tyr réplique en se proclamant métropole de Sidon; puis, sous Auguste, selon Strabon, les deux cités se disputent le titre de métropole de la Phénicie, qu'Achille Tatius donne toujours à Sidon au $\mathrm{II}^{\mathrm{e}}$ siècle p.C., alors que les monnaies de Tyr le portent déjà sous Domitien ${ }^{42}$. Si l'affrontement par types et légendes monétaires interposés semble tourner à l'avantage des Tyriens au cours du $\mathrm{III}^{\mathrm{e}}$ siècle $p . C$., c'est uniquement en raison de l'arrêt du monnayage de leurs rivaux après le règne de Sévère Alexandre (222-235).

\section{L'intégration de la lignée cadméenne dans les cultes phéniciens}

Les généalogies de Cadmos conservent précisément le souvenir de la rivalité entre les Tyriens et les Sidoniens, soucieux les uns et les autres d'établir une parenté légendaire entre les villes grecques et leurs cités respectives : Agénor, prince descendant d'une dynastie argienne et père du premier roi thébain, règne tantôt sur les premiers, tantôt sur les seconds. Si ces relations mythiques contradictoires s'expriment de manière différente à Tyr et à Sidon, elles se manifestent par l'intégration de la lignée cadméenne dans les cultes de chacune des deux cités.

Les témoignages concordants de l'historien Arrien et du poète Nonnos de Panopolis montrent qu'Agénor était honoré d'un culte à Tyr. Selon Nonnos, à son

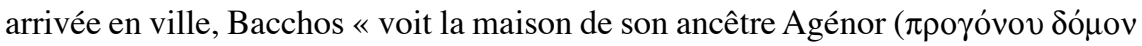
$\varepsilon \hat{i} \delta \varepsilon v$ 'A $\gamma$ ńvopos), il découvre le palais et l'appartement de Cadmos ; et il pénètre dans la chambre virginale mal gardée de la jeune Europe, jadis enlevée, et se souvient de son père Zeus porteur de cornes ${ }^{43}$. Dans son étude sur les Dionysiaques, Pierre Chuvin estime pour sa part que les monuments cités sont fictifs ${ }^{44}$. Il est vrai que le long passage lyrique que Nonnos consacre à la description de la ville

41. C'est ce que montre l'épigramme qui accompagne la dédicace officielle d'une statue érigée par les Sidoniens pour le juge Diotimos fils de Dionysios vers 200 a.C. Voir BIKERMAN 1939.

42. Strabon, Géographie 16, 2, 22. Pour Achille Tatius, Leucippé et Clitophon 1, 1, 1, Sidon reste la métropole de la Phénicie et son peuple est le père de celui de Thèbes. Cf. Sartre 2001 et Rey-Coquais 2001, sur le statut métropolitain de Tyr.

43. Nonnos de Panopolis, Dionysiaques 40, 356-359 (éd. et trad. Simon 1999, p. 176).

44. Chuvin 1991, p. 227. 
fleure bon le folklore poétique. Néanmoins, la demeure tyrienne d'Agénor chez le poète correspond chez Arrien à «ce qu'on appelle le sanctuaire d'Agénor »

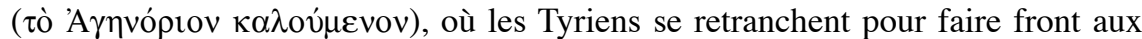
Macédoniens lors du siège de la ville par Alexandre ${ }^{45}$. L'existence de ce lieu saint paraît peu douteuse si l'on rappelle qu'Agénor est réputé être le fondateur de la cité $^{46}$. On lira à ce sujet l'historiette que rapportent plusieurs auteurs tardifs à la suite de Malalas, qui fait dériver le nom de la cité de celui de Tyrô, l'épouse d'Agénor ${ }^{47}$. L'invention de la nymphe éponyme de Tyr parachève la construction mythologique qui justifie l'appartenance des Tyriens à la lignée cadméenne.

À Sidon, c'est sur un abus de langage que semble reposer l'identification de la ville à la «maison des nobles Agénorides ${ }^{48}$. Pour fonder et promouvoir l'idée qu'Agénor avait régné sur leur cité et non sur celle de leurs voisins, les Sidoniens ont certainement tiré parti du fait que leur ethnique est le synonyme grec de Phénicien depuis l'époque archaïque. Ils ont pu s'appuyer sur l'autorité

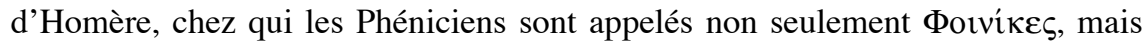

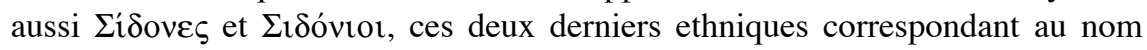
habituel des Phéniciens dans les textes assyriens et dans l'Ancien Testament. La tradition homérique a été reprise par les auteurs classiques : Euripide, par exemple, s'y conforme dans le prologue du Phrixos, qui chante la geste du fils d'Agénor, Cadmos, « ayant quitté jadis la ville sidonienne » pour gagner Thèbes, même s'il paraît s'en séparer lorsqu'il présente Europe comme une Tyrienne dans son Hypsipyle ${ }^{49}$. L'origine sidonienne de la lignée cadméenne découlerait de

45. Arrien, Anabase 2, 24, 2. Bikerman 1939, p. 94, et LightFoot 2003, p. 298, situent à tort ce sanctuaire à Sidon.

46. Quinte-Curce, Histoires 4, 4, 19, où Tyr est «fondée par Agénor » (condita ab Agenore).

47. Malalas, Chronographie 2, 7 (éd. Thurn 2000, p. 22-23) ; Chronicon paschale, p. 76 (éd. Dindorf 1832, t. 1) ; Jean d'Antioche, Chronique, fr. 6, 15 (FHG 4, p. 544) ; Georges Harmatolos, Chronicon breve 1, 9, 3 (PG 110, col. 57D-60A); Georges Cedrenus, Compendium historiarum 38 ( $P G$ 121, col. 65C) ; Eustathe, Commentaire à Denys le Périégète 911 (GGM 2, p. 375). Cf. BüHLER 1968, p. 8-9, sur les textes attribuant à Europe une autre mère que Tyrô.

48. L'expression est attestée par la dédicace d'époque hellénistique qu'étudie BIKERMAN 1939 : Diotimos y est honoré comme le premier de ses concitoyens à avoir apporté la

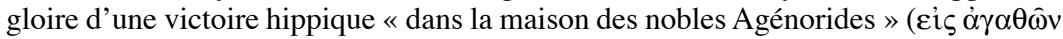

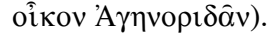

49. On comparera, chez Euripide, Hypsipyle, fr. 1, 3, 1. 21-22 (KANNICHT 2004, fr. 752g) au prologue du Phrixos, cité par Aristophane, Grenouilles 1225-1226 (KanNicht 2004, fr. 819, 1. 1-2), et connu par le P. Oxy. 2455, 1. 268-269 (Kannicht 2004, p. 861-862); cf. également Euripide, Bacchantes 170-172, où Cadmos vient d'une cité sidonienne. 
l'interprétation littérale de cette tradition, à une époque difficile à déterminer ${ }^{50}$. Quoi qu'il en soit, l'ambiguïté sur le lieu où Cadmos et les membres de sa famille se sont établis en Phénicie paraît totale à partir du ${ }^{\mathrm{er}}$ siècle $a . C$., en particulier chez les poètes, d'Ovide à Nonnos de Panopolis, où le lecteur peine parfois à distinguer Tyrien de Sidonien ${ }^{51}$. Même si des raisons de métrique ont pu déterminer le choix de l'un ou l'autre des deux qualificatifs, je me demande s'il ne faut pas considérer que seul l'usage de Sidonien prête à confusion, contrairement à celui de Tyrien: Sidonien aurait deux lectures, soit « Sidonien » soit « Phénicien », alors que Tyrien ne s'interpréterait que comme «Tyrien ». Cette proposition permet de résoudre la contradiction apparente entre les passages où plusieurs auteurs tardifs présentent Agénor comme le fondateur de Tyr et ceux où ils le mettent en relation avec les Sidoniens ${ }^{52}$. L'établissement de la lignée cadméenne à Sidon est finalement admis comme une évidence au $\mathrm{II}^{\mathrm{e}}$ siècle p.C. : d'une part, les monnaies de la cité se reconnaissent alors au fait que l'enlèvement d'Europe y est représenté ${ }^{53}$; d'autre part, à la même époque, le cliché passe dans le roman d'Achille Tatius, qui décrit un tableau vu à Sidon, où figure Europe sur le taureau nageant au bord du rivage de la cité phénicienne ${ }^{54}$. Bien plus tard, au vi ${ }^{\mathrm{e}}$ siècle $p . C$., sur le pavement qui représente l'enlèvement d'Europe dans la mosaïque de Sarrîn en Osrhoène, une inscription située à gauche de la représentation schématique d'une ville localise encore et toujours l'épisode au même endroit ${ }^{55}$.

À l'époque hellénistique, les relations mythiques entre les cités phéniciennes et leurs parentes grecques pouvaient se prolonger dans l'actualité par l'admission

50. BÜHLER 1968, p. 10, considère Publius Nigidius Figulus, érudit néopythagoricien du temps de Varron, comme le premier auteur chez qui l'origine sidonienne d'Europe est attestée. Sans s'en expliquer, il ne tient donc pas compte d'un fragment attribué à Hellanicos par JАСов, FGrHist 1 A, n 4, p. 121 (Boiotika, fr. 51), qui fait d'Europe une fille de Phœnix, comme dans l'Iliade 14, 321, tout en ajoutant qu'elle est originaire de Sidon. Il est vrai que ce fragment est tiré d'une scholie à l'Iliade qui résume en même temps Hellanicos et Apollodore, Bibliothèque 3, 21-25, de sorte que l'information qu'il transmet pourrait n'être qu'une glose tardive et donc négligeable dans la recherche des sources les plus anciennes sur l'origine sidonienne d'Europe.

51. Ovide, Fastes 5, 603-618 ; Sénèque, Edipe 713-717 et Hercule furieux 9; Stace, Thébaïde 1, 5-16. Cf. BüHLER 1968, p. 10. Selon CHuvin 1991, p. 247, les qualificatifs Sidonien et Tyrien sont interchangeables dans le poème de Nonnos de Panopolis.

52. Malalas, Chronographie 2, 7 et 5, 4 (éd. Thurn 2000, p. 22-23 et 70) ; Chronicon paschale, p. 47 et 76 (éd. Dindorf 1832, t. 1).

53. De Dea Syria 4 (éd. Lightfoot 2003, p. 248).

54. Achille Tatius, Leucippé et Clitophon 1, 1, 2-13.

55. Balty 1990, p. 9-10, 47-50, 96, pl. A et 20-21. 
d'un Phénicien aux grands concours panhelléniques ${ }^{56}$. Sous l'Empire romain, la référence constante à ces relations vise toujours à glorifier la patrie dans la haine du voisin, mais elle se traduit aussi par des questionnements sur l'identité des grands dieux poliades. À Sidon même, au milieu du II siècle p.C., les discussions qui ont cours en ville au sujet des traditions locales prennent la forme de débats théologiques sur l'interprétation de la grande déesse poliade : l'auteur du De Dea Syria rapporte ainsi l'opinion d'un prêtre du sanctuaire sidonien d'Astarté selon laquelle ce lieu de culte est en fait dédié à Europe, sœur de Cadmos et fille du roi Agénor, avant de préciser que tout le monde ne s'accorde pas sur ce point dans la cité ${ }^{57}$. Ce témoignage fait écho à celui de Quinte-Curce : au moment où Alexandre assiège Tyr, les Sidoniens sauvent des Tyriens en souvenir de leur parenté «car on croyait», précise l'historien latin comme s'il manifestait son scepticisme, «qu'Agénor avait fondé les deux villes » ${ }^{58}$. Plus généralement, on opposera les propos du prêtre sidonien d'Astarté, pour qui la déesse qu'il sert n'est autre qu'Europe, à ceux d'un Philon de Byblos, partisan d'un patriotisme panphénicien farouchement opposé à l'interprétation grecque des dieux locaux : ces prises de position représentent les deux réactions extrêmes à l'assimilation des divinités helléniques et orientales.

\section{Conclusion}

Le mythe de la lignée cadméenne, à laquelle Ino-Leucothéa et son fils appartiennent, a été adapté à Tyr et à Sidon au cours de l'époque hellénistique, où il a pu être sollicité comme argument d'autorité, à titre de preuve d'une parenté des citoyens de chacune des deux villes avec ceux des vénérables cités d'Argos et de Thèbes. Il n'y a pas à s'étonner de retrouver ici les règles tacites qui déterminent habituellement l'élaboration des mythes de parenté entre cités. En revanche, il est moins banal de constater que la réception locale de ces récits s'accompagne de l'attribution de théonymes grecs à des divinités locales et de l'introduction de nouveaux héros grecs dans le panthéon officiel de Tyr et de Sidon jusqu'à susciter

56. BIKERMAN 1939, p. 95 : «Les relations mythiques se prolongent dans l'actualité. Diotimos, un Phénicien, peut faire courir ses chevaux aux jeux Néméens vers 200 parce qu'un prince d'Argos régna jadis sur Sidon ». Plus généralement, à propos des parentés légendaires entre cités, voir CURTY 1995 ; sur ce livre, qui ne tient pas compte des relations entre Sidon, Tyr, Argos et Thèbes, car il se fonde principalement

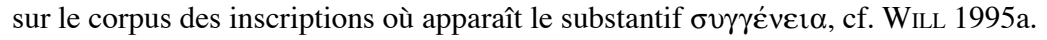

57. De Dea Syria 4 (éd. LightFoot 2003, p. 248). L'image d'Europe apparaît dans un temple sur une monnaie frappée à Sidon sous Élagabal : il pourrait s'agir d'une idole ou d'un relief cultuel. Voir Price \& TRell 1977, p. 156-157 ; cf. p. 13, fig. 277.

58. Quinte-Curce, Histoires 4, 4, 15 : quippe utramque urbem Agenorem condidisse credebant. 
des discussions théologiques parmi les fidèles. Dans cette perspective, tout l'intérêt des monuments découverts dans l'arrière-pays des villes phéniciennes est aussi de montrer que les prétentions de leurs citoyens sont corrélées à l'époque romaine à la pratique effective d'un culte rendu à Leucothéa et à Mélicerte sur les confins montagneux de leur cité. Même si les cultes ruraux et urbains pourraient avoir pour point commun le caractère oraculaire de Leucothéa et de Mélicerte, évident à Haloua et plausible à Tyr, ces monuments doivent toutefois être considérés comme l'expression originale, propre au milieu montagnard, des rapprochements établis en ville entre les dieux de la Grèce et les divinités locales.

Julien AliQuot

Doctorant à l'Université de Tours Maison de l'Orient et de la Méditerranée

HISOMA, UMR 5189 (Lyon)

Antenne de Tours

\section{Bibliographie}

Aliquot 2002

J. Aliquot, « Leucothéa de Segeira », Syria 79, p. 231-248.

Aliquot 2004

J. AliQuot, «Inscriptions grecques et antiquités de Haloua », BAAL 8, p. 301-314.

BALTY 1981

J.-C. Balty, « Agreus I », LIMC 1/1, p. 306.

BALTY 1990

J. Balty, La mosaïque de Sarrîn (Osrhoène), Beyrouth.

BIKERMAN 1939

É. BIKERMAn, « Sur une inscription grecque de Sidon », dans Mélanges syriens offerts

à Monsieur René Dussaud, Paris, p. 91-99.

BLATTER 1984

R. BLATTER, «Argonautai », LIMC 2/1, p. 591-599.

BONNET 1986

C. Bonnet, « Le culte de Leucothéa et de Mélicerte, en Grèce, au Proche-Orient et en Italie », SMSR 52, p. 53-71.

BONNET 1988

C. Bonnet, Melqart, Louvain/Namur.

BONNET 1991

C. Bonnet, «Tinnit, sœur cadette d'Astarté ? À propos des cultes de Deir el-Qal'a près de Beyrouth », WO 22, p. 73-84. 
BONNET 1996

C. BonNet, Astarté, Rome.

BONNET 1997

C. BonNET, «De l'histoire des mentalités à l'histoire des religions : à propos de Leucothéa et de trois petits cochons », SEL 14, p. 91-104.

BÜHLER 1968

W. BÜHLER, Europa, Munich.

Chambry 1933

É. Chambry, Lucien de Samosate. Euvres complètes, traduction nouvelle avec notices et notes 1 , Paris.

\section{Сне́HAB 1962}

M. CHÉHAB, « Tyr à l'époque romaine. Aspects de la cité à la lumière des textes et des fouilles », MUSJ 38, p. 11-40.

Chuvin 1991

P. Chuvin, Mythologie et géographie dionysiaques, Clermont-Ferrand.

CIL 3 Suppl.

A. von Domaszewski, O. HiRschFeld \& T. Mommsen, Corpus inscriptionum latinarum 3. Inscriptiones Orientis et Illyrici latinarum supplementum, Berlin (1902).

Clermont-Ganneau 1898

C. Clermont-Ganneau, «Le culte de la déesse Leucothea dans la région de l'Hermon », Recueil d'archéologie orientale 2, Paris, p. 98-101.

CURTY 1995

O. CURTY, Les parentés légendaires entre les cités grecques, Genève.

DINDORF 1832

L. Dindorf (éd.), Chronicon paschale, ad exemplar Vaticanum, Bonn.

\section{FGrHist}

F. JАCOBY (éd.), Die Fragmente der griechischen Historiker, Leyde (1923-1954).

$F H G$

C. MülLER (éd.), Fragmenta Historicorum graecorum, Paris (1853-1870).

GARNAUd 1991

J.-P. Garnaud (éd.), Achille Tatius d'Alexandrie. Le roman de Leucippé et Clitophon, Paris.

GATIER 1982

P.-L. GATIER, «Inscriptions religieuses de Gérasa », ADAJ 26, p. 269-275.

GATIER 1994

P.-L. GATIER, «Objets consacrés de la Syrie romaine », dans Y. LE Bohec (éd.), L'Afrique, la Gaule, la Religion à l'époque romaine. Mélanges à la mémoire de Marcel Le Glay, Bruxelles, p. 774-779. 
GEBHARD 2005

E.R. Gebhard, « Rites for Melikertes-Palaimon in the Early Roman Corinthia », dans S.J. Friesen \& D.N. Schowalter (éd.), Urban Religion in Roman Corinth, Cambridge (Mass.), p. 165-203.

$G G M$

C. MüLler (éd.), Geographi Graeci Minores, Paris (1855-1861).

GHADBAn 1981

C. GHADBAn, «Les frontières du territoire d'Héliopolis-Baalbeck à la lumière de nouveaux documents », dans T. FAHD (éd.), La géographie administrative et politique d'Alexandre à Mahomet, Strasbourg, p. 143-168.

HAJJAR 1985

Y. HaJJAR, La triade d'Héliopolis-Baalbek 3, Montréal.

HAJJAR 1990

Y. HaJJAR, «Dieux et cultes non héliopolitains de la Béqa', de l'Hermon et de l'Abilène à l'époque romaine », ANRW 2, 18.4, p. 2509-2604.

JONES 1986

C.P. Jones, Culture and Society in Lucian, Cambridge (Mass.)/Londres.

KANNICHT 2004

R. Kannicht (éd.), Tragicorum Graecorum Fragmenta 5. Euripides, Göttingen.

Lefebvre \& Perdrizet 1919

G. Lefebvre \& P. Perdrizet, Les graffites grecs du Memnonion d'Abydos, Nancy/ Paris/Strasbourg.

LighTFOOT 2003

J.L. Lightfoot (éd.), Lucian. On the Syrian Goddess, Oxford.

MACLEOD 1961

M.D. MACLEOD (éd.), Lucian VII, Cambridge (Mass.)/Londres.

MACLEOD 1987

M.D. MACLEOD (éd.), Luciani opera 4. Libelli 69-86, Oxford.

MESHORER 1984-1985

Y. Meshorer, « The Coins of Caesarea Paneas », INJ 8, p. 37-58.

MichON 1905

É. Michon, «Antiquités gréco-romaines provenant de Syrie conservées au Musée du Louvre », RBi, p. 564-578.

Millar 1993

F. Millar, The Roman Near East (31 BC-AD 337), Cambridge (Mass.)/Londres.

MOUTERDE 1942-1943

R. Mouterde, « Monuments et inscriptions de Syrie et du Liban », MUSJ 25, p. 2379.

NASTER 1986

P. NASTER, «Ambrosiai petrai dans les textes et sur les monnaies de Tyr », dans

C. Bonnet et alii (éd.), Religio Phoenicia, Namur, p. 361-371. 
Patillon \& Segonds 1995

M. Patillon \& A.P. Segonds (éd.), Porphyre. De l'abstinence 3, Livre IV, Paris.

PERDRIZET 1903

P. PerdRIzet, «Une inscription d'Antioche qui reproduit un oracle d'Alexandre d'Abonotichos », CRAI, p. 62-66.

PERDRIZET 1932

P. PERDRIZET, «Légendes babyloniennes dans les Métamorphoses d'Ovide », RHR 105, p. 193-228.

PIÉRART 1998

M. Piérart, «Panthéon et hellénisation de la colonie romaine de Corinthe: la "redécouverte" du culte de Palaimon à l'Isthme », Kernos 11, p. 85-109.

PORAT 1997

P. PorAT, «A Fragmentary Greek Inscription from Tel Jezreel », Tel Aviv 24, p. 167168.

PRICE \& TRELl 1977

M.J. Price \& B.L. Trell, Coins and their cities, Londres.

Rey-Coquais 1999

J.-P. ReY-CoQuaIS, « Deir el Qalaa », Topoi 9/2, p. 607-628.

Rey-CoQuais 2001

J.-P. Rey-CoQuaIs, «Jordanie, d'Alexandre à Moawiya : un millénaire d'hellénisation », Studies in the History and Archaeology of Jordan 7, p. 359-364.

ROBERT 1980

L. RoBert, À travers l'Asie Mineure, Athènes/Paris.

SARTRE 1993

M. SARTRE, «Faits divers et histoire des mentalités : à propos de quelques noyés et de trois petits cochons », Syria 70, p. 51-67.

SARTRE 2001

M. SARTRE, «Les manifestations du culte impérial dans les provinces syriennes et en Arabie », dans C. Evers \& A. Tsingarida (éd.), Rome et ses provinces. Genèse $\&$ diffusion d'une image du pouvoir. Hommages à Jean-Charles Balty, Bruxelles, p. $167-186$.

SARTRE 2003

M. SARTRE, D’Alexandre à Zénobie, $2^{2}$ éd., Paris.

SEYRIG 1963

H. SEYrIG, «Antiquités syriennes. 83. Les grands dieux de Tyr à l'époque romaine », Syria 40, p. 19-28 (Seyrig 1966, p. 121-130).

SEYRIG 1966

H. SEYRIG, Antiquités syriennes 6, Paris.

SIMON 1999

B. Simon (éd.), Nonnos de Panopolis. Les Dionysiaques 14, Chants XXXVIII-XL, Paris. 
TALBOT 1912

E. Talbot, Euvres complètes de Lucien de Samosate. Traduction nouvelle, $6^{\mathrm{e}}$ éd., Paris.

THURn 2000

I. Thurn (éd.), Ioannis Malalae Chronographia, Berlin.

VIKELA 1994

E. Vikela, Die Weihreliefs aus dem Athener Pankrates-Heiligtum am Ilissos, Mayence.

VIKELA \& VOLLKOMMER 1992

E. Vikela \& R. VolLKommeR, « Melikertes », LIMC 6/1, p. 437-444.

WALTZ 1960

P. Waltz (éd.), Anthologie grecque 3, 2éd., Paris.

WEST 1997

M.L. West, The East Face of Helicon. West Asiatic Elements in Greek Poetry and Myth, Oxford.

WILL 1995a

Éd. WILL, « Syngeneia, oikeiotès, philia », RPh 69, p. 299-325.

WILL 1950-1951

Er. WILL, « Au sanctuaire d'Héraclès à Tyr : l'olivier enflammé, les stèles et les roches ambrosiennes », Berytus 10, p. 1-12 (WILl 1995b, p. 243-255).

WILL 1995b

Er. WILL, De l'Euphrate au Rhin, Beyrouth. 


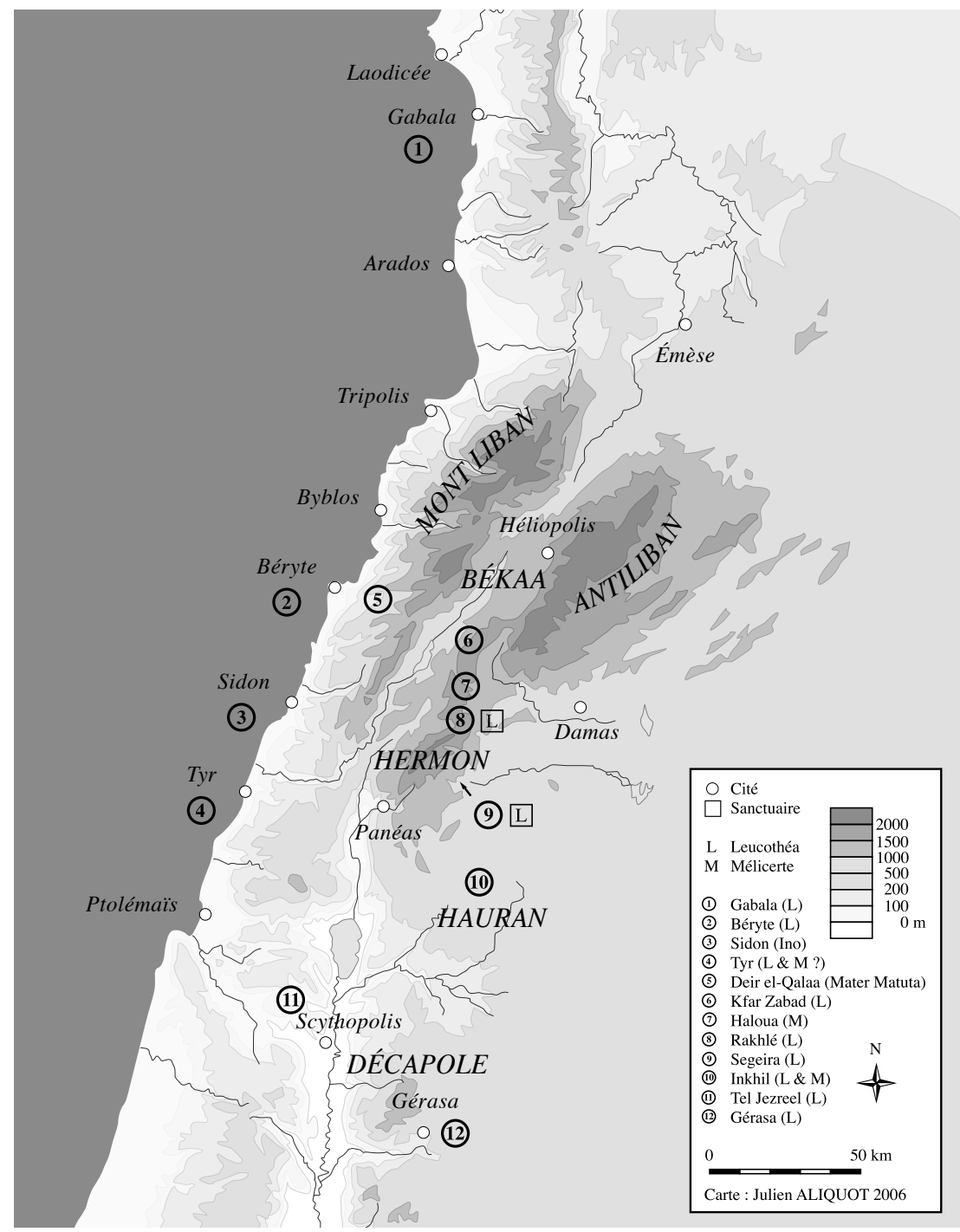

Leucothéa et Mélicerte au Proche-Orient 